\title{
Prediction of MACE by Angiographic Perfusion Score (APS) in Risk Stratification following Percutaneous Coronary Stenting in STEMI without Thrombolytic Therapy
}

KHALED MD IQBAL, SYED ALI AHSAN, MD MUKHLESUR RAHMAN, MSI TIPU CHOWDHURY, KHURSHED AHMED, TANJIMA PARVIN, ABU BAQAR MD JAMIL, MD. FAKHRUL ISLAM KHALED, SABINDRA BHUPAL MALLA, PRITAM KUMAR GACHCHHADAR, MRM MANDAL

\author{
Department of Cardiology, Bangabandhu Sheikh Mujib Medical University (BSMMU), Dhaka \\ Address of correspondence: Dr. Khaled Mohammad Iqbal, Resident, Department of Cardiology, BSMMU, Dhaka. \\ Email : rimjim0990@gmail.com
}

\begin{abstract}
:
Background: ST-elevation myocardial infarction (STEMI) is a major cause of mortality worldwide. PCI remain gold standard management of STEMI. An Angiographic Perfusion Score (APS) is a combination of TIMI Flow Grades (TFG) and myocardial perfusion (TMPG) grades before and after PCI. So it can accurately measure both epicardailand myocardial perfusion and predict major adverse cardiac events (MACE).

The aim of the study is to evaluate the association of APS for determining the short term clinical outcomes after PCI in ST-segment elevation myocardial infarction without thrombolytic therapy.

Methodology: This cross sectional observational study carried out among adult patients presenting STsegment elevation myocardial infarction without thrombolytic pretreatment to the cardiology department of BSMMU who underwent Percutaneous Coronary Intervention and met inclusion and exclusion criteria. This study was conducted from April 2017 to March 2018. After informed written consent data collection was carried out by using a data sheet. Calculated sample size of the study was 205. Angiogram was done by SIEMENS AXOM ARTIS machine, CD Analysis to see TFG \& TMPG for calculation of APS was done by two specialist observer (Professor, Associate or Assistant Professor) MACE was observed within 30 days. Chi square test was done to see the association of APS with MACE by Statistical Package for Social Science program 20 version of computer on the basis of different variables.

Results: MACE distribution of the study patients revealed that only $7.8 \%$ of the patients had MACE whereas 92.2\% of the patients had no MACE among 205 patient APS score with MACE distribution of the study patients revealed that $9.3 \%$ patient of partial perfusion group and $44.4 \%$ patient of failed perfusion group had MACE but there were no major adverse cardiac event in full perfusion group of patient. The difference were statistically highly significant $(p<0.05)$ among three groups but there were no differences in the baseline characteristics of those who had full, partial or failed perfusion of APS.

Conclusion: APS which combines TFG with TMPG before and after PCI is a better discriminator of 30 day MACE than TMPG or TFG alone taken only after PCI. It can identify high risk patients who need implementation of early invasive strategies. We conclude that APS is a better predictor of MACE to take care of patient more accurately.
\end{abstract}

Key wards: APS, MACE.

University Heart Journal 2018; 14(2): 16-21

Introduction:

ST-segment elevation myocardial infarction (STEMI) results from a disruption of a vulnerable coronary atherosclerotic plaque complicated by intraluminal thrombus formation, embolization and variable degrees of coronary obstruction. Patients with total or near total occlusion may present with acute ST-Elevation
Myocardial Infarction (STEMI) requiring emergency reperfusion therapy. ${ }^{1}$

Angiographic Perfusion Score (APS) is proposed as a simple score which links epicardial and myocardial perfusion parameters before and after percutaneous coronary intervention (PCI), is a predictor of short term outcome in patients with ST-segment elevation 
myocardial infarction (STEMI) treated with PCI. ${ }^{2}$ Percutaneus coronary intervention (PCI) is a preferred method of reperfusion for ST-segment Elevation Myocardial Infarction (STEMI). ${ }^{3}$

The aim of Reperfusion therapy in acute myocardial infarction (AMI) is early and sustained reperfusion of the myocardium at risk. Reperfusion therapy is considered to be angiographically successful when a good TIMI (thrombolysis in myocardial infarction) flow is achieved in the infarct-related coronary artery. ${ }^{4,5}$ Improved epicardial flow assessed by TIMI flow grades (TFG) has been related to reduced mortality after coronary revascularization. ${ }^{4,6,7,8}$ However, even when a good TIMI flow is achieved, some patients have less than optimal reperfusion at the tissue level, and myocardial reperfusion is not always achieved in patients with a good TIMI flow of epicardial coronary artery. Several mechanisms have been suggested to be involved such as no reflow and distal embolization.., 10

TIMI myocardial perfusion grade (TMPG) is an angiographic measure of myocardial perfusion at capillary level. ${ }^{9}$ TMPG has been found to be useful in both pharmacological and catheter based reperfusion after AMI and is suggested as a useful indicator of successful myocardial reperfusion. ${ }^{10}$ Patients with both normal epicardial flow and myocardial perfusion have been shown to have a very low mortality rate of $0.73 \% .{ }^{11}$ Thus, the TMPG adds additional prognostic information to the conventional epicardial TFG. ${ }^{11}$ Incorporation of these two variables envisaging a combined index of epicardial and myocardial microvascular blood flow has been suggested as the Angiographic Perfusion Score (APS). ${ }^{12}$ APS is a simple, angiographic metric that takes into account indices of epicardial and myocardial perfusion, both before and after PCI, to arrive at a single perfusion grade. The APS is the sum of the TFG (0-3) added to the TMPG (0-3) before and after PCI, therefore, a total grade of 0 to 12 is possible. Failed perfusion was defined as an APS of 0-3, partial perfusion as an APS of 4-9, and full perfusion as an APS of 10-12. Among STEMI patients with larger infarct sizes, the association of APS with the incidence of death or MI has been found to be statistically significant with an APS score of (0-9). ${ }^{12}$

Evidence thus favors that the APS, which combines grades of epicardial and tissue level perfusion before and after
PCI may be closely associated with infarct size, and may prove valuable in clinical risk stratification. The aim of this study is to assess short term clinical outcome after PCI in a patient of ST-segment elevation myocardial infarction (STEMI) without thrombolytic pretreatment.

\section{Materials And Methods}

\section{Place of study:}

Cross-sectional observational study was done in the Department of Cardiology, University Cardiac Center, Bangabandhu Sheikh Mujib Medical University, Shahbag, Dhaka from July 2017 to June 2018.

\section{Study population:}

Adult patients present with ST-segment elevation myocardial infarction (STEMI) without thrombolytic pretreatment who are undergoing Percutaneous Coronary Intervention (PCI).

The eligibility criteria was as follows:

\section{Inclusion Criteria:}

1) STEMI patients undergoing Primary PCI.

2) STEMI patient presenting after 12 hours with cardiogenic shock.

3) STEMIpatient with ongoing symptom presenting within 72 hours.

\section{Exclusion Criteria}

1) STEMI patients treated with thrombolytic.

2) STEMI presented after 72 hours.

3) Failed PCI in STEMI patients.

4) PCI in NSTEMI, UA patients

A data sheet was used for each patient. Data were analyzed by SPSS 20.0 software

\section{Results:}

Figure no. 01 reveals that majority (34.1\%) of the patients belonged to age group of 51-60 years, $32.2 \%$ of them belonged to age group of 41-50 years and only $13.9 \%$ belonged to the age group of d" 40 years. The mean age and SD was found $52.83 \pm 10.147$ years.

The following pie chart reveals that among all, $88.8 \%$ of the patients were male and only $11.2 \%$ of the patients were female.

Interpretation : Most of the patient were male which correlate with the trend of coronary artery disease which is a male predominant disease. 


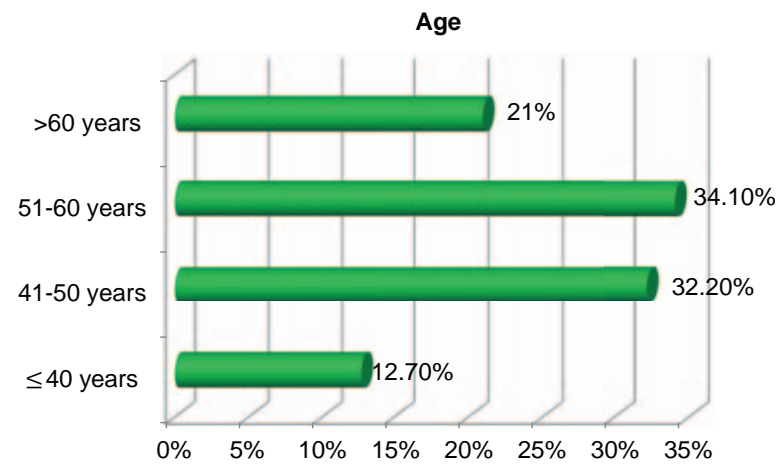

Fig.-1: Distribution of the respondents according to age $(n=205)$

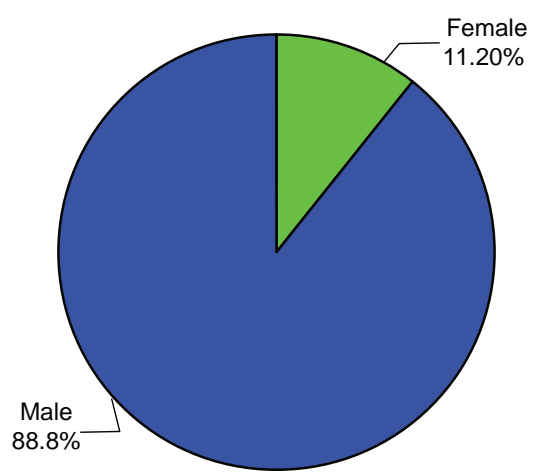

Fig.-II: Distribution of the respondents according to $\operatorname{sex}(n=205)$

\section{Table-I}

Presence of conventional risk factors in study population

\begin{tabular}{lccc}
\hline Characteristics & Frequency & Percent & Mean \pm SD \\
\hline BMI & 17 & 8.3 & $27.482 \pm 2.4429$ \\
$18.5-24.9$ (Normal) & 165 & 80.5 & \\
$25-29.9$ (Over weight) & 23 & 11.2 & \\
$>30$ (Obese) & & 65.4 & 34.6 \\
Smoker & 134 & & \\
Yes & 71 & 86.3 \\
No & & 13.7 \\
Hypertension & 177 & & \\
Yes & 28 & 63.4 \\
No & & 36.6 & \\
Diabetes Mellitus & 130 & 25.4 \\
Yes & 75 & 74.6 & \\
No & & 87.8 \\
F/O of CAD & 52 & 12.2 \\
Yes & 153 & \\
No & & 6.3 \\
Dyslipidaemia & 192 & 93.7 \\
Yes & 13 & \\
No & & 98.0 & \\
Past history of MI & 13 &
\end{tabular}

Table I reveals presence of conventional risk factors in study population. It was observed among 205 patients. (80.5\%) patients were overweight BMI(25-29.9). Among them $65.4 \%$ were smoker, $86.3 \%$ were hypertensive, $63.4 \%$ were diabetic, $93.6 \%, \%$ had Dyslipidaemia, $25.4 \%, 6.3 \%$ and $2 \%$ hadF/O of CAD, Past history of MI and Past history of coronary intervention respectively. 
Table-II

Frequency distribution of APS score $(n=205)$

\begin{tabular}{lcc}
\hline APS & Frequency & Percentage \\
\hline Full & 67 & 32.7 \\
Partial & 129 & 62.9 \\
Failed & 9 & 4.4 \\
\hline
\end{tabular}

Table II shows that majority (62.9\%) of the patients had partial perfusion, $32.7 \%$ had full perfusion and rest of the $4.4 \%$ had failed perfusion.

Table-III

Frequency distribution ofMACE $(n=205)$

\begin{tabular}{lcc}
\hline MACE & Frequency & Percentage \\
\hline Yes & 16 & 7.8 \\
No & 189 & 92.2 \\
\hline
\end{tabular}

Table 3 disclose that MACE distribution of the study patients, It was observed that only $7.8 \%$ of the patients had MACE, whereas $92.2 \%$ of the patients had no MACE.

Table 4 finds that Chi square test between APS score with MACE distribution of the study patients. It was observed that the frequency of MACE in the patients with full perfusion was $0.0 \%, 9.3 \%$ of partial perfusion and $44.4 \%$ among failed perfusion group. The difference were statistically highly significant $(\mathrm{p}<0.05)$ among three groups.

Table $-\mathrm{V}$ : MACE distribution in perfusion status of patients disclose that out of the 16 with routine procedures, there were no MACE at complete reperfusion group. In patients with Partial reperfusion MACE were $12(9.3 \%)$, among them $1(0.8 \%)$ death, 2(1.5\%) QMI, 5(3.9\%) non QMI \& 4(3.1\%) TVR respectively whereas among $4(44.4 \%)$ patient with failed perfusion death was 2(22.2\%), non QMI was $1(11.11 \%)$ and QMI was 1(11.11\%).

Table-IV

Chi square test between APS score with MACE

\begin{tabular}{|c|c|c|c|c|c|c|c|c|}
\hline \multirow[t]{3}{*}{ MACE score } & \multicolumn{6}{|c|}{ APS } & \multirow{3}{*}{ Chi square } & \multirow{3}{*}{$P$ value } \\
\hline & \multicolumn{2}{|c|}{ Full $(\mathrm{n}=67)$} & \multicolumn{2}{|c|}{ Partial $(n=129)$} & \multicolumn{2}{|c|}{ Failed $(\mathrm{n}=9)$} & & \\
\hline & $\mathrm{N}$ & $\%$ & $\mathrm{~N}$ & $\%$ & $\mathrm{~N}$ & $\%$ & & \\
\hline Yes & 0 & 0 & 12 & 9.3 & 4 & 44.4 & & \\
\hline No & 67 & 100.0 & 117 & 90.7 & 5 & 55.6 & 61.85 & $0.001^{\mathrm{s}}$ \\
\hline Total & 67 & 100.0 & 129 & 100.0 & 9 & 100.0 & & \\
\hline
\end{tabular}

$\mathrm{P}$ value reached from chi square test

$\mathrm{s}=$ Significant

Table-V

Association between MACE in the APS group (Outcome within 30 days) ( $n=16)$

\begin{tabular}{|c|c|c|c|c|c|}
\hline \multirow[t]{2}{*}{$\overline{\mathrm{MACE}}$} & \multicolumn{4}{|c|}{ APS } & \multirow[b]{2}{*}{ P value } \\
\hline & $\begin{array}{c}\text { Full }(\mathrm{n}=67) \\
\mathrm{n}(\%)\end{array}$ & $\begin{array}{c}\text { Partial }(\mathrm{n}=129) \\
\mathrm{n}(\%)\end{array}$ & $\begin{array}{c}\text { Failed }(n=9) \\
n(\%)\end{array}$ & $\begin{array}{c}\text { Total }(\mathrm{n}=205) \\
\mathrm{n}(\%)\end{array}$ & \\
\hline \multicolumn{6}{|l|}{ Death } \\
\hline Yes & 0 & $1(0.8 \%)$ & $2(22.2 \%)$ & $3(1.5 \%)$ & 0.01 \\
\hline No & $67(100 \%)$ & $128(99.2 \%)$ & $7(77.8 \%)$ & $202(98.5 \%)$ & \\
\hline \multicolumn{6}{|l|}{ QMI } \\
\hline Yes & $0(0.0 \%)$ & $2(1.5 \%)$ & $1(11.1 \%)$ & $3(1.5 \%)$ & 0.55 \\
\hline No & $67(100 \%)$ & $127(98.4 \%)$ & $9(100 \%)$ & $202(98.5 \%)$ & \\
\hline \multicolumn{6}{|l|}{ Non QMI } \\
\hline Yes & $0(0.0 \%)$ & $5(3.9 \%)$ & $1(11.11 \%)$ & $6(2.9 \%)$ & 0.55 \\
\hline No & $67(100 \%)$ & $124(96.1 \%)$ & $8(88.89 \%)$ & $198(97.1 \%)$ & \\
\hline \multicolumn{6}{|l|}{ TVR } \\
\hline Yes & $0(0.0 \%)$ & $4(3.1 \%)$ & $0(0.0 \%)$ & $4(1.9 \%)$ & 0.30 \\
\hline No & $67(100 \%)$ & $125(96.9 \%)$ & $9(100 \%)$ & $201(98.1 \%)$ & \\
\hline Composite $(n=16)$ & $0(0.0 \%)$ & $12(13.9 \%)$ & $4(44.4 \%)$ & $22(10.7 \%)$ & \\
\hline
\end{tabular}

$\mathrm{S}=$ significant

ns $=$ not significant 


\section{Discussion:}

This study was under taken to evaluate the association of APS for determining the short term clinical outcomes after PCI in ST-segment elevation myocardial infarction without thrombolytic therapy. This study sample size was 205 STEMI patients who underwent PCI.

Majority (62.9\%) of the patients had partial reperfusion, $32.7 \%$ had full reperfusion and rest of the $4.4 \%$ had failed reperfusion. It was observed that only $7.8 \%$ of the patients had MACE, whereas $92.2 \%$ of the patients had no MACE.

It was observed that MACE in the patients who had full perfusion was $0.0 \%, 9.3 \%$ in partial perfusion group and $44.4 \%$ in failed perfusion group respectively. The difference were statistically highly significant $(\mathrm{p}<0.05)$ among three groups. A previous study similar with present study conducted by ${ }^{14}$, Narain et al., on Prognostic value of Angiographic Perfusion Score (APS) following percutaneous interventions in acute coronary syndromes. APS identified as a significant tool of reperfusion status than TMPG or TFG alone. A significantly lower incidence of MACE was observed in the full perfusion group as compared to the failed perfusion group.

\section{Clinical Outcomes:}

\section{Adverse outcome within 30 days}

According to the present study, it was observed that $15.6 \% \& 13.2 \%$ of the patients had Post PCI Angina \& minor bleeding respectively at 30 days While $8.8 \%$ had vascular access site complication, 2.9\% had non QMI, $2.4 \%$ had fatal arrhythmia, $1.9 \%$ had TVR, death was $1.5 \%$ and $1.5 \%$ of the patients had QMI.

MACE distribution in perfusion status of patients:

Out of the 16 with routine procedures, there were no MACE at complete reperfusion group. In patient with Partial reperfusion were $12(9.3 \%)$ among them $1(0.8 \%)$ death, 2(1.5\%) QMI, 5(3.9\%) non QMI, 4(3.1\%) \& TVR respectively. Patient with failed perfusion there were MACE in 4 patients (44.4\%) among them death was 2(22.2\%), non QMI was 1(11.11\%), QMI 1(11.11\%) respectively.

There were no differences in the baseline characteristics of those who had full, partial or failed perfusion.

\section{Conclusion:}

The APS combines grades of epicardial and tissue level perfusion before and after PCI to arrive at a single angiographic predictor of MACE. No patients with full angiographic perfusion died but partial or full angiographic perfusion group of patients are associated with MACE. To optimize outcomes, both epicardial and myocardial perfusion must be restored to normal. TFG which is the favored method of predicting outcomes has the ambiguity of using a single parameter taken at one point in time, and is low on sensitivity to identify patients with good prognosis after PCI. This can be overcome by the simple scoring system of APS which combines TMPG with TFG before and after PCI andtakes care of the minor differences arising out of operator related interpretation of the angiographic parameters. APS is a better discriminator of 30 day MACE than TMPG or TFG alonetaken only after PCI. We conclude that APS is a better predictor of MACE to take care of patient more accurately.

\section{References:}

1. Agudas, SC., Estratifi, T., de Risco.'Acute Coronary Syndrome: Treatment and Risk Stratification'.RevistaBrasileira de Terapia intensive. 2008;20(7): 197-204

2. Brzozowska-Czarnek, A., Urbanik, A., Rakowski, T., Krakow, PL.2012, 'Is Angiographic perfusion score assessed in patients with acute myocardial infarction correlated with cardiac magnetic resonance infarct size and $\mathrm{N}$-terminal pro-brain natriuretic peptide in 6-onth follow up'.ECR 2012.

3. Rakowski, T., Legutko, J., Kleczynski, P., Brzozowska-Czarnek, A., Dziewierz, A., Siudak, Z., 'Angiographic perfusion score assessed in patients with acute myocardial infarction is correlated with cardiac magnetic resonanceinfarct size and NterminalProbrain natriuretic peptide in 6 month followup'. Journal of Thrombosis and Thrombolysis. 2010; 30: 441-45

4. The TIMI study group, 'The thrombolysis in myocardial infarction (TIMI) trial. Phase 1 findings'. N Engl J Med. . 2015;312: 932-36.

5. Wikipedia, the free encyclopedia (2018) TIMI. [online] available from https://en. wikipedia.org/wiki/TIMI

6. Simes R.J, Topol E.J \& Holmes D.R (1995) Link between the angiographic substudy and mortality outcomes in a large randomized trial of myocardial reperfusion: importance of early and complete infarct artery reperfusion. Circulation.91:1923-28.

7. Vogt A., Von Essen R., Tebbe U (1993) Impact of early perfusion status of the infarct-related artery on short-term mortality after thrombolysis for acute myocardial infarction: retrospective analysis of four German multicenter studies. J Am CollCardiol. 21:1391-95.

8. Karagounis L., Sorensen S.G., Menlove R.I (1992) Does thrombolysis in myocardial infarction TIMI perfusion grade 2 represent a mostly patent artery or a mostly occluded artery? Enzymatic and electrocardiographic evidence from the TEAM-2 study.J Am CollCardiol.17:1-10. 6.

9. Anderson J.L., Karagounis L.A., Becker L.C. TIMI perfusion grade 3 but not grade 2 results in improved outcome after thrombolysis for myocardial infarction: ventriculographic, enzymatic, and electrocardiographic evidence from the TEAM-3 study. Circulation.1993;87:1829-39. 
10. Gibson C.M., Cannon C.P., Daley W.L. The TIMI frame count: a quantitative method of assessing coronary artery flow. Circulation.1996;93:879-88.

11. Gibson,CM., Cannon, CP., Murphy, SA., 'Relationship of the TIMI myocardial perfusion grades, flow grades, frame count and percutaneous coronary intervention to long-termoutcomes after thrombolytic administration in acutemyocardial infarction'.Circulation. 2002; 105: 1909-13.

12. Gibson MC \& Schömig A (2004) Coronary and Myocardial Angiography. Angiographic Assessment of Both Epicardial and Myocardial Perfusion. Circulation;109: 3096-3105. [online] available fromhttp://circ. ahajournals.org/content/109/25/3096
13. Gibson CM, Murphy SA, Morrow DA, Aroesty JM, Gibbons RJ, Gourlay SG, Barron HV, Giugliano RP, Antman EM, Braunwald E (2004) Angiographic perfusion score: an angiographic variable that integrates both epicardial and tissue level perfusion before and after facilitated percutaneous coronary intervention in acute myocardial infarction. Am Heart J;148(2):336-40. [online] available from https://www.ncbi.nlm.nih.gov/pubmed/15309006

14. Narain VS, Fischer L, Puri A, Sethi, R \&.Dwivedi, SK 2013 (janfeb), 'Prognostic value of Angiographic Perfusion Score (APS) following percutaneous interventions in acute coronary syndromes.'Indian Heart J,Vol. 65, Issue 1, Pages 1-6. [online] available from https://www.sciencedirect.com/science/article/pii/ S0019483212003215. 